\title{
Notes on Global Mean-Square Values of the Geomagnetic Field and Secular Variation
}

\author{
Heikki NeVANLINNA
}

University of Helsinki, Department of Geophysics, Fabianinkatu 24, SF-00100 Helsinki 10, Finland

(Received August 25, 1986; Revised October 23, 1986)

\begin{abstract}
The spatial power spectrum of the geomagnetic field was analyzed by calculating the spectrum for the $X, Y$ and $Z$ components separately. Based on different slopes of the power spectrum lines it was concluded that the dipole field originates partly from the surface layer of the outer core partly from the inner core. The dipole field from the inner core is axially symmetric.

A new method was introduced for separating the drifting and non-drifting parts in the spatial power spectrum of secular variation. Equations were derived which connect the non-drifting part with the energy density spectrum.

The non-drifting part has been rather stable since 1965. In the drifting part the harmonic constituents $n=1$ and $n=4$ have increased roughly by a factor five during the last 20 years.
\end{abstract}

\section{Introduction}

The mean-square value $W(n)$ of the total magnetic field over the Earth's surface for each harmonic $n$ is given by

$$
W(n)=(n+1) \sum_{m=0}^{n}\left\{\left(g_{n}^{m}\right)^{2}+\left(h_{n}^{m}\right)^{2}\right\}
$$

where $g_{n}^{m}$ and $h_{n}^{m}$ are the Schmidt normalized Gaussian coefficients of a spherical harmonic expansion (CHAPMAN and BARTELS, 1940). Equation (1) was first introduced by MAUERSBERGER (1956) and LUCKE (1957) and later used by LOWES $(1966,1974)$ and JAMES $(1968)$. Physically $W(n)$ represents the mean energy density of the geomagnetic field because if multiplied by the factor $1 / 2 \mu_{0}\left(\mu_{0}\right.$ is the vacuum permeability) it has the dimension of $\mathrm{J} / \mathrm{m}^{2}$ (see e.g. MEYER et al., 1983; MEYER, 1985). $W(n)$ has been called "Lowes parameter" by MALIN et al. (1983) and KERRIDGE and BARRACLOUGH (1985) in their studies on the global jerk phenomenon around the year 1970 .

There are two formulae similar to Eq. (1) related to the secular variation. The first one is:

$$
\dot{W}(n)=2(n+1) \sum_{m=0}^{n}\left(g_{n}^{m} \dot{g}_{n}^{m}+h_{n}^{m} h_{n}^{m}\right)
$$


which is the time derivative of $W(n)$ (MEYER et al., 1983). In this equation the dots represent the one year change in the Gaussian coefficients and in the mean-square field. The second formula is:

$$
Q(n)=(n+1) \sum_{m=0}^{n}\left\{\left(\dot{g}_{n}^{m}\right)^{2}+\left(h_{n}^{m}\right)^{2}\right\}
$$

which gives the mean-square of the secular variation field (LOWES, 1974; ALLDREDGE, 1986).

Plots of $\lg (W)$ and $\lg (|\dot{W}|)$, (here $\lg$ symbolizes $\log _{10}$ ) have been used to determine the radius $\left(R_{\mathrm{c}}\right)$ of the magnetic source layer in the core. The slope, $-2 \cdot \lg \left(R_{\mathrm{c}} / R\right),(R$ is the radius of the Earth), of the linear regression line through the points $W(n), n=2, \ldots, 10-12$, (in Eq. (1)) determines this radius. As has been pointed out by many authors (e.g. LOWES, 1974; MEYER et al., 1983), the dipole term $(n=1)$ does not fit to this line indicating that the dipole field is caused by deeper sources than other harmonics. The points of $W(n)$ for which $n>14$, represent magnetic sources in the crust of the Earth (LOWES, 1974; CAIN et al., 1974; MEYER et al., 1983).

MEYER et al. (1983) have shown that the slope of $\lg (|\dot{W}|)$ versus $n$ gives the same radius for the source layer as calculated from Eq. (1). In this case, the dipole term lies on the same line as the higher order terms indicating that the secular variation of the dipole field is originated in the same layer at the core-mantle boundary. In both cases this radius is roughly $150 \mathrm{~km}$ shorter than the radius of the core determined by seismic methods.

However, when $Q(n)$ is plotted against $n$, it decreases much more slowly than the corresponding values of $\dot{W}(n)$. The reason, as pointed out by LOWES (1974) is due to the westward drift of the non-dipole field pattern which causes a substantial part of the secular variation.

In this paper I will present a new quantitative method for separating the drifting and non-drifting part from the secular variation spectrum $Q(n)$. Some comments on the sources of the dipole part in the energy spectrum of the main field will be given.

\section{The Dipole Part in the Energy Density Spectrum of the Main Field}

Figure 1a shows the mean energy density spectrum $W(n)$ as calculated from the IGRF 1980.0 (PEDDIE, 1982). The slope of a straight line obtained from a leastsquares fit to the points $n=2, \ldots, 10$ gives $3300 \mathrm{~km}$ as the radius of the core source layer. This is within $20 \mathrm{~km}$ the same as obtained by LOWES (1974), LANGEL and ESTES (1982) and MEYER et al. (1983). In Fig. 1a, it can be seen how the dipole field term $\lg W(1)$ lies clearly above the regression line determined by the rest of the harmonics. Using the same logic for the points $n=1$ and 2 as for the points $n=2, \ldots, 10$, we can draw a straight line through the first two harmonics which represent one part of spatial magnetic spectrum due to sources deep in the core. The slope of this line gives $1160 \mathrm{~km}$ as the radius of the apparent source shell which is close to the value $(1200 \mathrm{~km})$ commonly accepted to be the radius of the inner core. 
Figure $1 \mathrm{~b}$ shows the mean-square values for each component $X, Y$ and $Z$. These values can be calculated using the following formulae given by LOWES (1966) and JAMES (1968)

$$
\begin{aligned}
& W_{X}(n)=\sum_{m=0}^{n}\{n(n+1) /(2 n+1)-m / 2\}\left\{\left(g_{n}^{m}\right)^{2}+\left(h_{n}^{m}\right)^{2}\right\} \\
& W_{Y}(n)=\sum_{m=0}^{n}(m / 2)\left\{\left(g_{n}^{m}\right)^{2}+\left(h_{n}^{m}\right)^{2}\right\} \\
& W_{Z}(n)=\sum_{m=0}^{n}(n+1)^{2} /(2 n+1)\left\{\left(g_{n}^{m}\right)^{2}+\left(h_{n}^{m}\right)^{2}\right\} .
\end{aligned}
$$

As can be seen in Fig. 1b, the energy density spectrum of the regression line for $\lg \left(W_{X}\right)$ and $\lg \left(W_{Z}\right)$ behaves in a similar way as the total field line in Fig. 1a. The dipole part differs again clearly from the regression line. However, the spectrum of the east component is different: now the dipole part $\lg W_{Y}(1)$ is very close to the line calculated from higher harmonics. This means that the dipole part in the $Y$ field originates in the same top layer of the core as the magnetic field described by the harmonic terms $n=2, \ldots$, 10 in the $X$ and $Z$ fields.

We can thus conclude that the part of the dipole field which is tilted in respect to the Earth's rotation axis has its sources near the boundary between core and mantle. The part of the dipole field which is probably coming from the inner core is axially symmetric.

Figure 1c shows $\lg (W)$ plots of the magnetic field of Jupiter and Saturn as calculated using the Gaussian coefficients from spherical harmonic analyses by RUSSELL (1980) and CONNERNEY et al. (1982), respectively. Because these analyses include only the harmonic terms up to $n=3$ and are based on a quite limited amount of data, it is not possible to determine exactly the slope of $\lg (W)$ lines. However, the square of the dipole field of both planets is more than one decade greater than the corresponding values of the next two harmonics as is also the case in the Earth's magnetic field. This tendency may be an indication that a substantial part of planetary dipole field is originating deep in the planet's core.

3. Drifting and Non-Drifting Parts in the Mean-Square Field of Secular Variation

Non-drifting (ND) and drifting (D) parts in the Gaussian coefficients for the main field and secular variation have been studied by many workers e.g. WHITHAM (1958), YUKUTAKE and TACHINAKA (1968), JAMES (1969, 1970), YUKUTAKE (1985).

Starting from equations for ND and D spherical harmonic coefficients derived by WHITHAM (1958) and JAMES (1969) we can write for the ND case for each $n$ and $m$ : $\dot{g}_{\mathrm{ND}}=c g$ and $h_{\mathrm{ND}}=c h$ where $c$ is a common proportional coefficient. For a maximum contribution to secular variation, $(\dot{g}-c g)^{2}+(h-c h)^{2}$ is a minimum which will be attained when $c=\dot{W} / 2 W$. This leads to the following equations for the ND secular variation: 


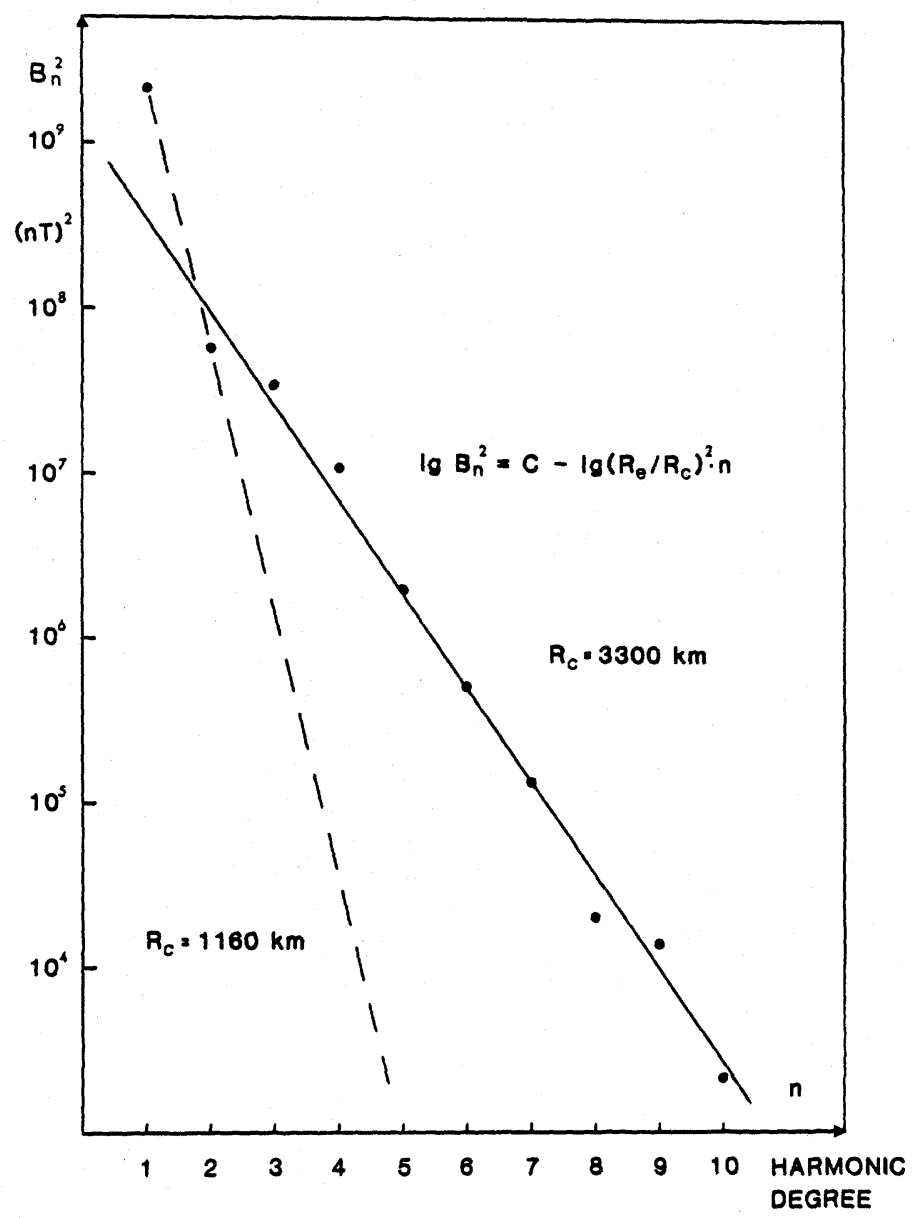

Fig. 1a. The mean-square of the total field $(B)$ as a function of the harmonic degree $(n)$ as calculated from the IGRF 1980 using Eq. (1). The slope of the regression line (solid line) gives the radius $(3300 \mathrm{~km})$ of the apparent source shell in the core. The dashed line drawn through the two first harmonics represents the spatial magnetic spectrum due to sources from the inner core.

$$
\dot{g}_{n, \mathrm{ND}}^{m}=\dot{W}(n) g_{n}^{m} / 2 W(n) ; \quad h_{n, \mathrm{ND}}^{m}=\dot{W}(n) h_{n}^{m} / 2 W(n)
$$

The drifting part which includes both the longitudinal and meridional components is

$$
\dot{g}_{n, \mathrm{D}}^{m}=\dot{g}_{n}^{m}-\dot{g}_{n, \mathrm{ND}}^{m} ; \quad h_{n, \mathrm{D}}^{m}=h_{n}^{m}-h_{n, \mathrm{ND}}^{m}
$$

By putting (4) and (4') to Eq. (2'), we get for ND and D parts of the mean-square of the secular variation field the following formulae: 


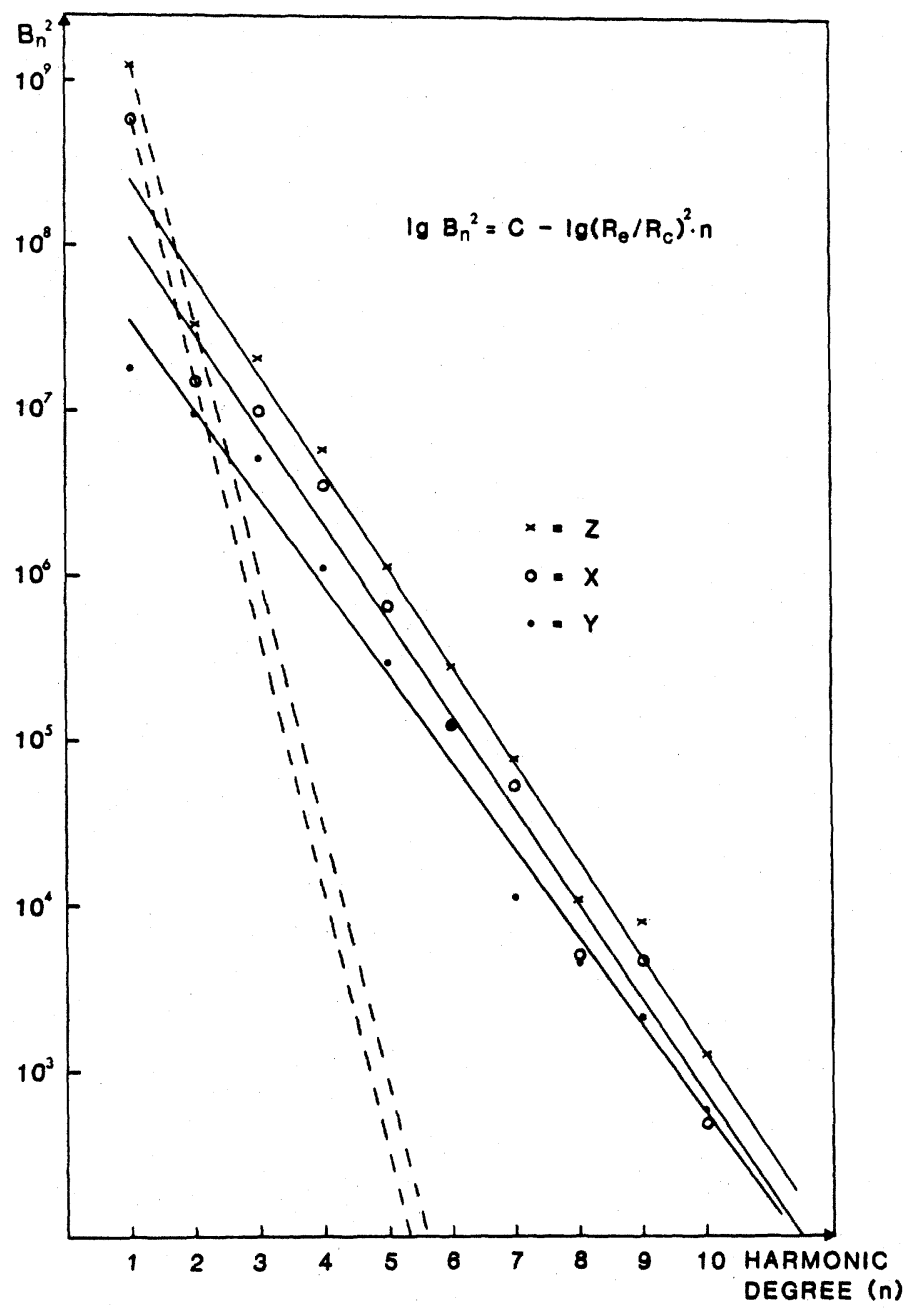

Fig. 1b. Same as Fig. 1a but the regression lines (solid lines) have been calculated separately for each component $(X, Y$ and $Z$ ) of the total field vector $B$. The dashed lines for $X$ and $Z$ demonstrate that part of the geomagnetic field which is supposed to originate in the inner core. In the case of the $Y$ component there seems to be no contribution from deeper part of the core because all harmonics fit rather well to the regression line which represents the spatial spectrum due to sources from the surface of the outer core.

$$
\begin{aligned}
& Q(n)_{\mathrm{ND}}=\dot{W}(n)^{2} / 4 W(n) \\
& Q(n)_{\mathrm{D}}=Q(n)-Q(n)_{\mathrm{ND}} .
\end{aligned}
$$




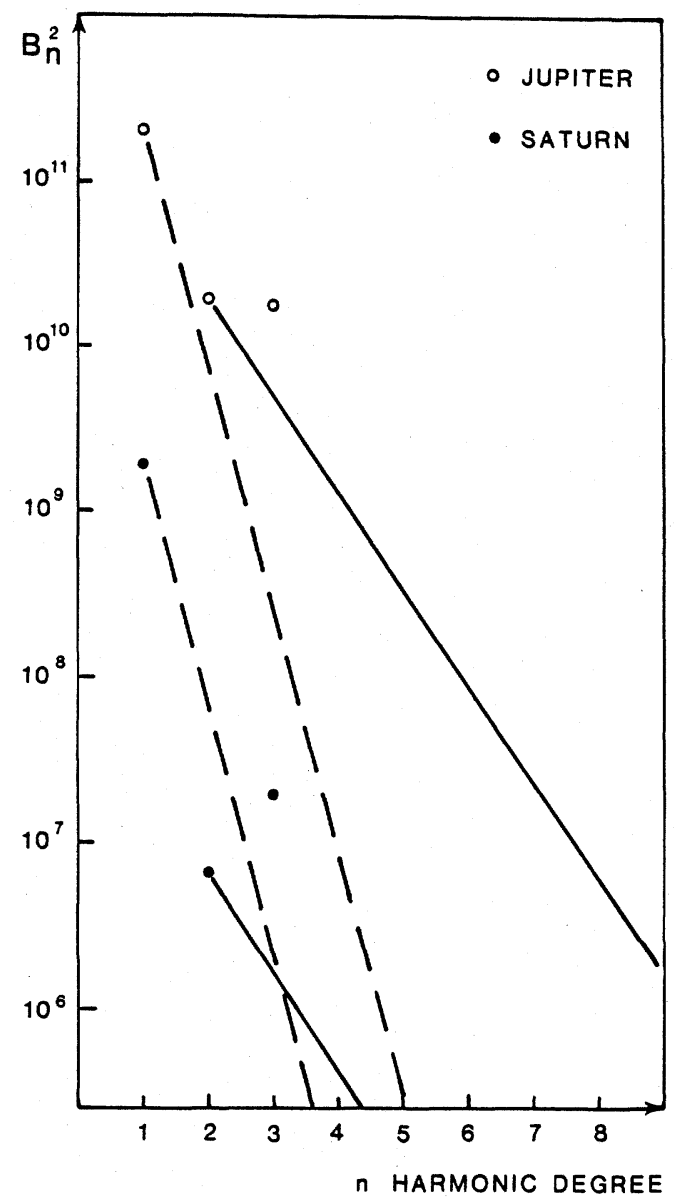

Fig. 1c. Same as Fig. 1a but calculated for the Planets Jupiter and Saturn using the Gaussian coefficients from spherical harmonic analyses by RUSSELL (1980) and CONNERNEY et al. (1982). The straight lines (solid and dashed) have exactly the same slope as those shown in Fig. 1a for the Earth's magnetic field but are shifted in the vertical direction.

However, if we try to separate the ND and D parts from the $\dot{W}(n)$ spectrum using Eqs. (4) and (4'), we get $\dot{W}(n)_{\mathrm{ND}}=\dot{W}(n)$ and $\dot{W}(n)_{\mathrm{D}}=0$. Thus the spectrum of the secular variation of the energy density $W(n)$ does not include contribution due to the drift of the field lines. This is of course expected because $W(n)$ is equal to the mean-square of the total field over the globe which remains unchanged in any movement of field patterns. Thus the spectrum $\dot{W}(n)$ is related only to the non-drifting intensity changes in the geomagnetic field. 
Figure 2 shows the ND and D parts in the mean-square secular field at five years interval from 1965 to 1985 using spherical harmonic coefficients compiled for the years from 1945 to 1985 by the IAGA DIVISION I WORKING GROUP 1 (1985). The secular variation terms were determined by taking the 10 years differences in the main field coefficients (the secular variation coefficients were given for 1985 by the Working Group).

We can see that these two parts of $Q(n)$ have a quite different distribution as function of $n$. The points of $\lg Q(n)_{\mathrm{ND}}$ up to $n=5$ fit rather well to the same straight line as in Fig. 1a. for the mean-square values of the main field $W(n), n=2, \ldots, 10$, and its time derivative $\dot{W}(n), n=1, \ldots, 10$. This is quite understandable because as shown by MEYER (1986) the regression lines of $\lg (W), \lg (|\dot{W}|)$ and $\lg (|\ddot{W}|)$ have always the same slope $(k)$ within a small error range. The slope of $\lg \left(Q_{\mathrm{ND}}\right)$ is also equal to $k$ because from Eq. (5) we get $\mathrm{d}\left\{\lg \left(\dot{W}^{2} / 4 W\right)\right\} / \mathrm{d} n=k$.

Higher harmonics $(n>5)$ in Fig. 2 scatter considerably but their absolute values are quite small, mostly below 1 in logarithmic units. The points for the drifting part of $Q$ decrease slower and are greater (except the dipole part) than the non-drifting values. Note that there is an r.m.s. secular variation field of order 1-3 nT/year at $n=10$ due to drift of the main field pattern but the non-drifting secular variation field has practically vanished when $n>7$.

Figure 2 also shows a comparison between $Q$ values calculated from spherical harmonic models and corresponding values obtained using a single dipole field model by ALLDREDGE (1984). In his paper a dipole was located at $r=0.45$ having both longitudinal and meridional drift velocity $0.2^{\circ} /$ year. As can be seen in Fig. 2, the shape of the $\lg \left(Q_{\mathrm{D}}\right)$ curve from the Alldredge's dipole model corresponds quite well to that calculated from spherical harmonic models. Note that the maximum in the $\lg \left(Q_{\mathrm{D}}\right)$ curve around $n=2-3$ predicted by the dipole model seems also to exist in the harmonic field model. This maximum can also be seen in the $\lg \left(Q_{\mathrm{ND}}\right)$ curves but it is not so pronounced.

The $Q$ values calculated for different epochs reveal some interesting trends in the secular variation field. Firstly we can see that the ND part of the secular variation spectrum have been rather stable during the last 20 years (except the higher harmonics but their scattering values may be due to inaccuracies in secular variation data). On the other hand the drifting part of the spectrum has changed rapidly. Since 1965 the dipole and the harmonic constituent $n=4$ (which was called by WINCH (1967) the sedecimupole field) of the secular variation spectrum have increased by a factor of 7 and 3.5, respectively. In the dipole field the term $h$ is now 6 times greater than 1965 . This recent trend in the dipole field is consistent with predictions obtained from the harmonic models based on long-term changes in the geomagnetic field (YUKUTAKE, 1985).

\section{Conclusions}

By studying the spatial spectrum of the mean-square values (or the mean energy density) of the total magnetic field and its components ( $X, Y$ and $Z$ ), it has been shown 


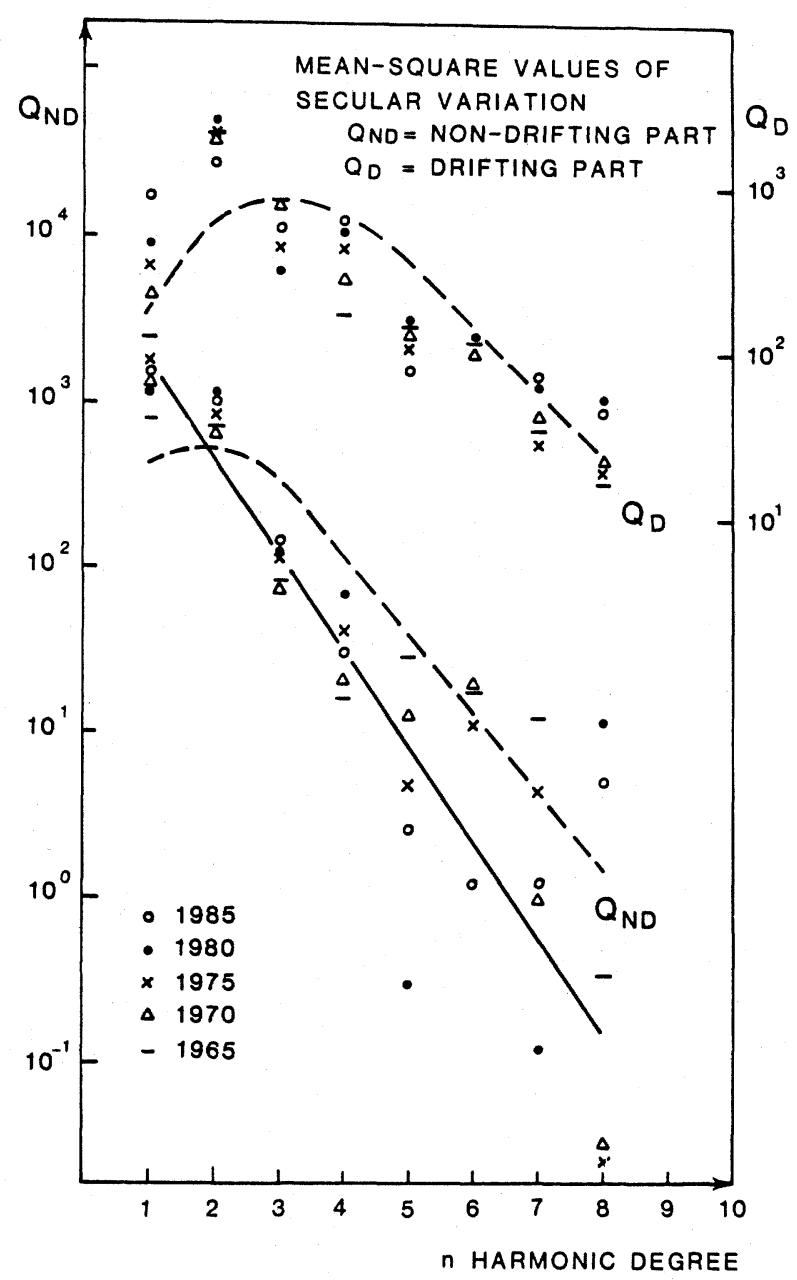

Fig. 2. The mean-square of the secular variation field $Q(n)$ as divided into drifting (D) and non-drifting (ND) parts from spherical harmonic models for 5 epochs from 1965 to 1985 (IAGA Division I WORKING GRouP 1, 1985). The dashed lines are the $Q$ values taken from ALLDREDGE's (1984) single dipole model in which the dipole is locating at $r=0.45$ and the drift velocity is $0.2^{\circ} /$ year. The solid line in the lower part is the same line as for the mean-square value of the main field in Fig. 1a.

that a major part of the dipole field and some of the quadrupole field which are axially symmetric, seem to originate deep in the core. The same conclusion may be drawn from the magnetic energy density spectra for Jupiter and Saturn. The radius of the apparent core shell found here is roughly the same as the radius of the inner core.

Two different spectra have been used for the secular variation studies: the time derivative, $\dot{W}(n)$, of the energy density spectrum $W(n)$ (MEYER et al., 1983; MEYER, 
$1985,1986)$ and the mean-square values, $Q(n)$, of the secular variation field (LOWES, 1974; ALLDREDGE, 1984, 1986). These two spectra are quite different. $\dot{W}(n)$, when plotted in a logarithmic scale against the harmonic degree $n$, is linear and the slope gives as the radius of the apparent source layer the same value (c. $3000 \mathrm{~km}$ ) as is obtained from the main field spectrum. The spectrum $Q(n)$ decreases much more slower and is not linear when $n=1,2$. However, a separation of $Q(n)$ into the drifting and non-drifting parts shows that the spectrum of the latter part has the same linear character as $W(n)$ and $\dot{W}(n)$. On the other hand it was also shown that $\dot{W}(n)$ does not give a complete picture of the secular variation spectrum because it contains no information about secular variation due to the drift of the field patterns. Therefore, when studying secular variation using globally averaged values, $Q(n)$ is preferable because it has both the standing and drifting components.

During the last 20 years the drifting part of the secular variation spectrum has increased considerably more than the standing one. Especially the harmonic constituents $n=1$ and $n=4$ have intensified roughly fivefold.

\section{REFERENCES}

ALLDREDGE, L. R., Harmonics required in main field and secular variation models, J. Geomag. Geoelectr., 36, 63-72, 1984.

AlldRedGe, L. R., Comments on "Remark on the secular change in the energy density spectrum of the geomagnetic field" by Joachim Meyer, J. Geomag. Geoelectr., 38, 171-172, 1986.

Cain, J. C., W. M. Davis, and R. D. Regan, An $N=22$ model of the geomagnetic field, EOS, Trans. $A G U$, 56, 1108, 1974.

Chapman, S. and J. Bartels, Geomagnetism, Vol. 2, Oxford at the Clarendon Press, London, 1940.

Connerney, J. E. P., N. F. NESs, and M. H. Acuna, Zonal harmonic model of Saturn's magnetic field from Voyager 1 and 2 observations, Nature, 298, 44-46, 1982.

IAGA DIVISION I WORKING GROUP 1, International geomagnetic reference field revision 1985, J. Geomag. Geoelectr., 37, 1157-1163, 1985.

James, R. W., Multipole analysis. I. Theory, and geomagnetic multipoles 1965.0, Aust. J. Phys., 21, 455-464, 1968.

JAMES, R. W., Multipole analysis. II. Geomagnetic secular variation, Aust. J. Phys., 22, 481-495, 1969.

JAMES, R. W., Decomposition of geomagnetic secular variation into drifting and non-drifting components, J. Geomag. Geoelectr., 22, 241-252, 1970.

KERRIDGE, D. J. and D. R. BARRACLOUGH, Evidence for geomagnetic jerks from 1931 to 1971, Phys. Earth Planet. Inter., 39, 228-236, 1985.

LANGel, R. A. and R. H. Estes, A geomagnetic field spectrum, Geophys. Res. Lett., 9, 250-253, 1982.

LowES, F. J., Mean-square values on sphere of spherical harmonic vector fields, J. Geophys. Res., 71, 2179, 1966.

LowES, F. J., Spatial power spectrum of the main geomagnetic field, and extrapolation to the core, Geophys. J. R. Astr. Soc., 36, 717-730, 1974.

Lucke, O., Über Mittelwerte von Energiedichten der Kraftfelder. Wiss. Z. Päd. Hochschule Potsdam, Math.-Nat. Reihe, 3, 39-46, 1957.

MALin, S. R. C., B. M. Hodder, and D. R. BARRAClough, Geomagnetic secular variation: a jerk in 1970, Ebro Observatory 75th Anniversary Volume, 239-245, 1983.

Mauersberger, P., Das Mittel der Energiedichte des geomagnetischen Hauptfeldes an der Erdoberfläche und seine säkulare Änderung, Gerlands Beitr. Geophys., 65, 207-215, 1956.

MeYer, J., Secular variation of magnetic mean energy density at the source-layer depth, Phys. Earth Planet. Inter., 39, 288-292, 1985. 
MEYER, J., Remark on the secular change in the energy density spectrum of the geomagnetic field, $J$. Geomag. Geoelectr., 38, 165-170, 1986.

Meyer, J., J.-H. Hufen, M. Siebert, and A. Hahn, Investigations of the internal geomagnetic field by means of a global model of the Earth's crust, J. Geophys., 52, 71-84, 1983.

Peddie, N. W., International geomagnetic reference field: the third generation, J. Geomag. Geoelectr., 34, 309-326, 1982.

Russell, C. T., Planetary magnetism, Rev. Geophys. Space Phys., 18, 77-106, 1980.

Whiтнам, K., The relationship between the secular change and the nondipole fields, Can. J. Phys., 36, 1372-1396, 1958.

WINCH, D. E., The fourth order geomagnetic multipole: The sedecimupole, Pure and Appl. Geophys., 67, 112-122, 1967.

YUKUTAKE, T., A preliminary study on variations in the Gauss coefficients of the geomagnetic potential over several hundred years, Phys. Earth Planet. Inter., 39, 217-227, 1985.

Yukutake, T. and H. TACHINAKA, The non-dipole part of the Earth's magnetic field, Bull. Earthq. Res. Inst. Tokyo Univ., 46, 1027-1074, 1968. 